\title{
Comparing the performances of GARCH-type models in capturing cryptocurrencies volatility
}

\author{
ALESSANDRA AMENDOLA \\ University of Salerno \\ Dept. of Economics and Statistics \\ Via Giovanni Paolo II 132, Fisciano (SA) \\ ITALY \\ LUCA SENSINI \\ University of Salerno
}

Dept. of Management and Innovation System - Via Giovanni Paolo II 132, Fisciano (SA) ITALY

\begin{abstract}
The analysis of cryptocurrencies market behaviour is receiving significant attention from researchers and practitioners in the last decades. This paper aims at contributes to volatility estimations of the cryptocurrencies helping to highlight the main stylized facts and characteristics. The performance of different specifications of volatility modelling, within the GARCH class, have been compared through the Model Confidence Set (MCS) over four of the most capitalised cryptocurrencies, namely Bitcoin, Ethereum, Stellar and Ripple. Our empirical findings give evidence of strong asymmetric effects in cryptocurrencies volatility leading to a better performance of asymmetric GARCH specifications.
\end{abstract}

Key-Words: - Cryptocurrency; Volatility; GARCH; MCS

Received: January 4, 2020. Revised: June 3, 2020. Re-revised: June 8, 2020. Accepted: June 10, 2020.

Published: June 17, 2020

\section{Introduction}

Over the last few years, Bitcoin and several other cryptocurrencies have been launched aimed at addressing different features with various levels of adoption. It is not easy to give a generally accepted definition of the term cryptocurrencies [36]. In this paper, cryptocurrency is defined as a virtual currency not issued by a central bank, credit institution, or e- money institution, which can be used as a medium of exchange in an alternative to money in buying goods and services ([23], [24], [40] ). These cryptocurrencies, secured by a cryptography mechanism known as blockchain technology ([18],[7],[51]), may be traded at global level in multiple platform, to the extent that they are accepted. The blockchain is a public ledger, in which there is a record of any transaction completed using a cluster of computers linked in a peer- to-peer network [16]. This technology makes it difficult to counterfeit cryptocurrencies and also makes hard double spending. The pseudonym character, the low costs, the high-speed in transactions, the higher earnings prospects, the financial bubbles and the constant increase in available cryptocurrencies has determined a growing interest of the media, companies and researchers in this type of activity ([7], [41]). At the beginning of 2020, there were over 2500 cryptocurrencies (https://coinmarketcap.com). In recent years the accounting, eco- nomics and finance literature has intensified the study of cryptocurrencies, highlighting various critical issues. Some scholars have pointed out that cryptocurrencies actually perform the same functions as money (means of transactions, store of value and unit of ac- count) and therefore can be assimilated to money. However, this approach has been criticized as cryptocurrencies are not a generally accepted medium of exchange, like other currencies [42]. In addition, the high volatility of cryptocurrencies makes it difficult for investors to achieve stable returns or maintain value over time. According to other authors, only stable coins can effectively perform the functions of money, as it they have different technology and they have greater reliability for investors [54]. However, according to other authors, the stable coins are not sufficiently credible because pegged currencies and resources are not reliable [53]. Other research has shown that Bitcoin can be assimilated to commodities, such as gold, or to legal currencies, such as the dollar ([49], [22], [4]). Other studies have highlighted that Bitcoin may represent a safe haven asset and a valid tool to diversify the investment portfolio ([27], [52]). Conversely, other studies have shown that Ethereum, and Litecoin are more reliable investments than Bitcoin ([9], [10]) and this latter has poor reliability as a safe investment Smales:2019. Other authors highlighted that cryptocurrencies feature computer protocols that are out of any government control. In this regard, in the last few years, different trading 
venues have emerged that allow all traders to directly and continuously access the exchange less regulated than traditional exchanges. This circum- stance requires to pay maximum attention to how liquid they are these trading venues are [11]. In addition, cryptocurrencies are unregulated [14] and at- tract criminals, as they can use them to launder money, fund terrorism and buy illegal goods ([39],[26]). The above critical points indicate that the characteristics of cryptocurrencies are controversial. However, the main literature is substantially concordant that cryptocurrencies are extremely volatile and the main intent of most cryptocurrency transactions are attributable to speculations [[38],[15]] as confirmed by the evidence of price bubbles [[18],[29],[19],[28],[32]]. In this scenario, we have assisted to an increasing interest among researchers, investors, and regulators in investigating and analysing the cryptocurrencies market. The research contributions in this area were primarily devoted to the statistical analysis of Bitcoin, as the first decentralized cryptocurrency, (see [13], [35], [50]), and then, also given to the exponential growth that the cryptocurrency market has experienced in a relatively short time span leading to high prices fluctuation, the interest is moved to investigating and modelling the market behaviour and its volatility dynamics [22]. Starting from the seminal contribution by [25], volatility models has been extensively studied and, among them, Generalized Autoregressive Conditional Heteroskedasticity, GARCH, type models have grown to become the standard among academicians and practitioners [6] leading few authors to experience the use of such class of models into this context ([35], [17], [3], [48], [44], [46] among others). Each of the model's specification proposed in the literature enable to capture and highlights a peculiar characteristic of the market behaviour. However, given the large variety of volatility modelling options, model specification remains one of the main sources of uncertainty we need to deal with (c.f. [12], [37], [2]). In this paper, in light of these source of uncertainty, we evaluate and compare the performance of different models, within the GARCH class, in estimating and forecasting the volatility of cryptocurrencies by means of a Model Confidence Set (MCS) procedure as proposed by [33]. This aim is pursued through an extensive empirical evaluation considering a set of competing models consists of (symmetric and asymmetric) GARCH-type models applied to different cryptocurrencies, selected among the most capitalized ones and assuring data availability for the same time span. Namely, we consider Bitcoin, Ethereum, Stellar Lumens and Ripple, listed on the
New York Stock Exchange. Our main findings, whilst do not guarantee a straightforward preference among GARCH-type models, reveal that the asymmetric GARCH specification, and the Exponential GARCH model in particular, comes out as the most consistently best performing model.

The rest of the paper is organized as follows: Section 2 introduces the data considered; Section 3 gives some details on the methodology; Section 4 provides the empirical results. Finally, Section 5 concludes.

\section{Data and Preliminary analysis}

The analysis focus on daily cryptocurrency time series. The data of interest are daily log-differences on a sample period, selected based on the availability of quotations, spanning from the 1st of September 2015 to the 14th of February 2020 with a number of observations $n=1628$ collected from the Yahoo Finance provider. The starting period was chosen in or- der to include in the analysis some of the most relevant cryptocurrencies, such as Ethereum which is the second most valuable cryptocurrency, after Bitcoin, first launched in 2015. The four chosen cryptocurrencies exchanged in the New York Exchange are: Bitcoin (BTC), Ethereum (ETH), Stellar Lumens (XLM) and Ripple (XRP).

Bitcoin is the first virtual currency, created by Satoshi Nakamoto in 2008 to facilitate electronic payments between individuals, and it is the cryptocurrency market's leader. Bitcoin is based on an open permissionless blockchain: everybody can join or leave the public Bitcoin network at will. The creation of this currency takes place via a "mining" process and the aggregate number of Bitcoins that can be created through mining is limited: at any time points there will not exist more than 21 million bitcoins. The system is automated and limited by itself and doesn't require the intervention of a third authority, being free from government control and regulation. Out of all cryptocurrencies, Bitcoin is one of the easiest coins to be convert back into fiat currency and it can be promptly used as a medium of exchange in a large number of transactions. The limited number of Bit- coins, together with the fact that conversion rates for Bitcoins are determined by the market, results in high volatility in Bitcoins returns.

Ethereum, created by Vitalik Buterin in July 2015, is a decentralized platform that runs "smart contracts". Smart contracts are a digitalised version of traditional contracts or applications that run exactly as programmed which should enhance security and reduce the transaction costs that are related to contracting, without any possibility of censorship, fraud or third-party interference. Ethereum platform 
itself is not a cryptocurrency but, like other open and permisionless blockchains, it can also acts as a medium of exchange with the cryptocurrency Ether. Ether cryptocurrency can be converted into fiat currency on various cryptocurrency exchanges and is being accepted as a means of payment by a growing number of merchants.

Ripple, launched in 2012, is an open-source, P2P, decentralized digital payment platform focused on solving problems related to International Payment Transfers. Ripple launched the cryptocurrency XRP, that was built to become a bridge currency to allow financial institutions to settle cross-border payments faster and cheaper than using the traditional payment networks. Ripple can make an international money transfer within seconds and the fees are considerably lower when compared to what is charged by financial institutions and other cryptocurrencies. Unlike Bitcoin and Ethereum, of the total number of 100 billion XRP that will ever exist, 50 billion are owned by Ripple company. For this reason, Ripple is often criticized because the decentralization is the core idea behind cryptocurrencies. Ripple (XRP) makes use of its own specific consensus protocol and the total supply of XRP has been fully pre-mined by its inventors. Therefore, the company behind XRP, determines who may act as a transaction validator on its network. It runs on a permissioned blockchain, that is considered public, as it can be accessed and viewed by everybody.

Stellar, launched in 2014 by Jed McCaleb who is also the founder of Ripple, is an open-source distributed payments infrastructure and it can also be used to build smart contracts. The platform contributes to move money around the world and to conduct transactions between different currencies quickly and securely. Stellar has its own specific consensus protocol and use Lumens (XLM) to pay for transactions on the Stellar network. The network development is supported by Stellar Development Foundation. The goal of this organization is to contribute to the development of tools and social good initiatives around the Stellar network and financial inclusion. Stellar runs on a permissionless blockchain and if certain conditions are met: anyone can join the net- work at will and validate transactions without having to be pre-approved or verified by any central administrator. Lumens (XLM) can be directly converted into fiat currency through cryptocurrency exchanges but, at present, they are not developed yet if you compare them to the coins discussed above.

Tables 1 and 2 synthesize some summary statistics related to the daily exchange rates (versus the U.S. Dollar) levels and log-returns for the four considered cryptocurrencies.
It can be clearly observed that the values of exchange rates for Lumen and Ripple are significantly lower than that of Bitcoin and Ethereum for the whole period considered. At the time of writing, the exchange rate is approximately 0.06 USD to one Stellars and 0.23

Table 1: Summary statistics daily exchange rates: levels

\begin{tabular}{llllllc}
\hline & Mean & Median & SD & CV & Skew. & Kurt. \\
\hline BTC & 4649.6 & 3945.3 & 3993 & 0.8588 & 0.6607 & -0.2326 \\
\hline ETH & 206.52 & 155.47 & 240.51 & 1.646 & 1.8155 & 3.6380 \\
\hline XLM & 0.09317 & 0.04543 & 0.12565 & 1.3485 & 1.9715 & 4.6209 \\
\hline XRP & 0.27314 & 0.23616 & 0.3520 & 1.2887 & 3.4899 & 19.744 \\
\hline
\end{tabular}

Table 2: Summary statistics daily exchange rates: returns

\begin{tabular}{lllllll}
\hline & Mean & Median & SD & CV & Skew. & Kurt. \\
\hline BTC & 0.0023 & 0.0022 & 0.0385 & 16.519 & -0.104 & 4.5684 \\
\hline ETH & 0.0033 & -0.0006 & 0.0609 & 18.679 & 0.2343 & 4.0149 \\
\hline XLM & 0.0021 & -0.0008 & 0.0552 & 26.767 & 1.2075 & 11.977 \\
\hline XRP & 0.0023 & -0.0032 & 0.0686 & 29.953 & 3.1152 & 44.387
\end{tabular}

USD to one Ripple. In contrast, being the most capitalized cryptocurrency, Bitcoin has the largest values, which show its greater significance among cryptocurrencies. The series are positively skewed, apart from Bitcoin, with Ripple display the higher skewness values. As expected, all of them have an excess of kurtosis that is less evident for Bitcoin and Ethereum, quite high for Stellar and, even more evident, for Ripple. The patterns for levels and returns of the four cryptocurrencies are shown in Figures 1 and 2 respectively. The time plots show an increase of price level starting at the beginning of 2017 and the same as for the volatility.

\section{Modelling volatility with GARCH- type models}

Letting $r_{t}$ represent the log-return, that is, the first difference of the log-closing prices for day $t$, a General Conditional Heteroskedastic $(\mathrm{GCH})$ model can be defined as:

$$
\begin{gathered}
r_{t}=\sigma_{t} z_{t} \\
\sigma_{t}^{2}=\operatorname{var}\left(r_{t} \mid I^{t-1}\right)=f\left(I^{t-1} ; \boldsymbol{\theta}\right)
\end{gathered}
$$

where $z_{t}$ is a sequence i.i.d. random variables with zero mean and unit variance.

The GCH is a general structure that can include different specifications for conditional heteroskedasticity by different choices for the function $\mathrm{f}$ (.). Since the seminal contributions by [25] and [8] a huge number of models, in the GARCH class, have been proposed for the function $f($.$) . We$ move within this class of models considering symmetric and asymmetric GARCH specifications allow for different distributions of the error term, as detailed in the following. 

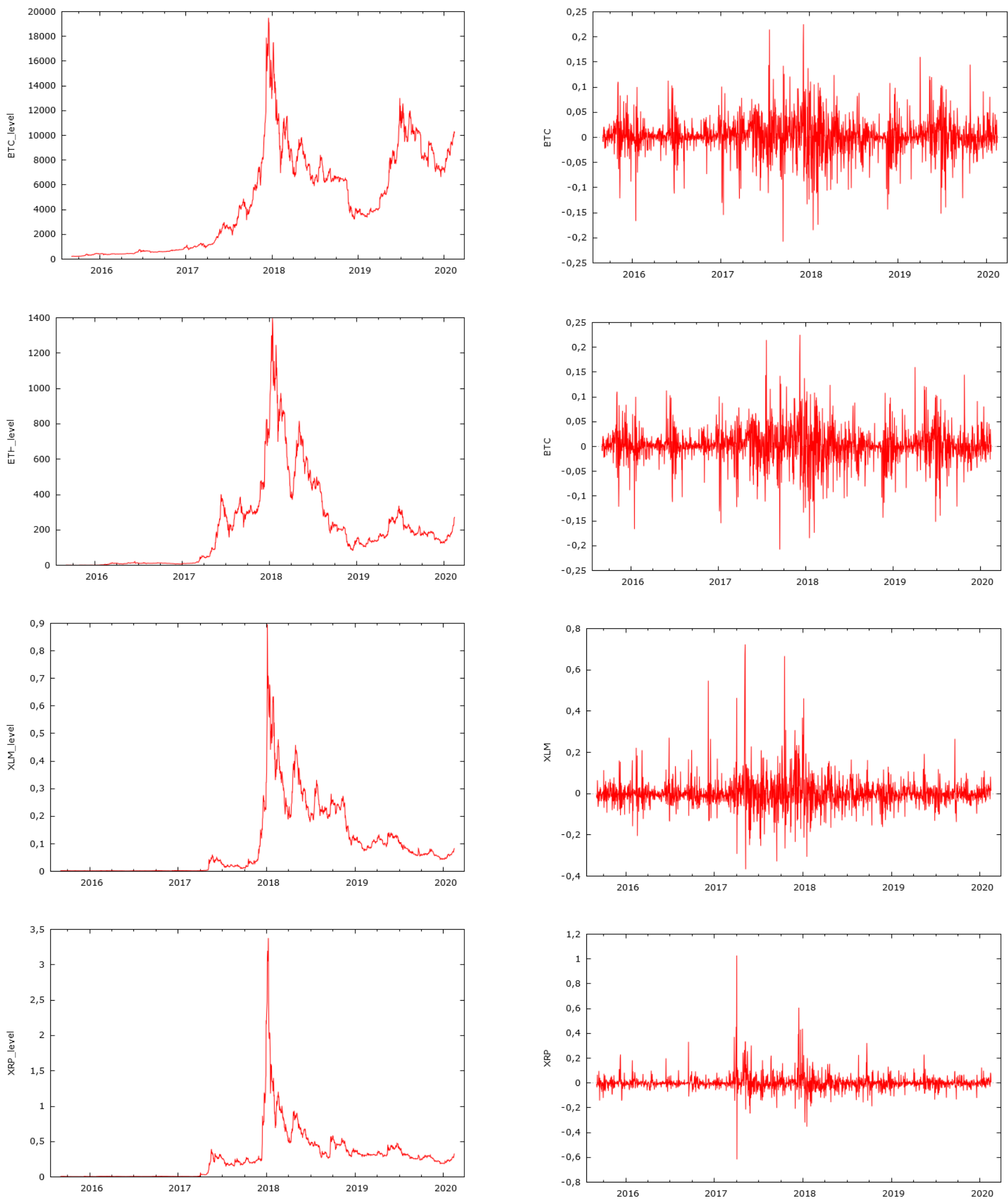

Figure 1: Cryptocurrencies daily exchange rates: levels time plots.

Figure 2: Cryptocurrencies daily exchange rates: returns time plots. 
For the $\operatorname{GARCH}(p, q)$ model proposed by [8] the conditional variance equation is given by:

$$
\sigma_{t}^{2}=\omega+\sum_{i=1}^{q} \alpha_{i} r_{i}^{2}+\sum_{j=1}^{p} \beta_{j} \sigma_{t-j}^{2}
$$

with $\quad \omega>0, \quad \alpha_{i} \geq 0, \beta_{j} \geq 0, \quad$ sufficient conditions for ensuring the positiveness of the conditional variance.

A model that can consider leverage effects is the Glosten-Jagannathan-Runkle GARCH (GJR) model proposed by [31]. In a GJR(p,q) dynamical equation for the conditional variance is given by:

$$
\begin{aligned}
\sigma_{t}^{2}= & \omega+\sum_{i=1}^{q} \alpha_{i} r_{i}^{2}+\sum_{j=1}^{p} \beta_{j} \sigma_{t-j}^{2}+ \\
& +\sum_{i=1}^{q} \gamma_{i} r_{t-i}^{2} I\left(r_{t-1}<0\right)
\end{aligned}
$$

where $I()$ is an indicator function equal to 1 if it is verified and 0 otherwise.

The asymmetric effect can be taken into account also with the Threshold GARCH model, $\operatorname{TGARCH}(p, q)$, proposed by [47] where the same dynamical equation is considered for modelling the standard deviation:

$$
\begin{aligned}
\sigma_{t}= & \omega+\sum_{i=1}^{q} \alpha_{i} r_{i}^{2}+\sum_{j=1}^{p} \beta_{j} \sigma_{t-j}+ \\
& +\sum_{i=1}^{q} \gamma_{i} r_{t-i}^{2} I\left(r_{t-1}<0\right) .
\end{aligned}
$$

In the Exponential GARCH model, $\operatorname{EGARCH}(p, q)$, proposed by [43], the volatility depends on both size and sign of lagged residuals and is given by the following equation of log-conditional variance:

$$
\begin{aligned}
\log \left(\sigma_{t}^{2}=\omega+\right. & \left.\sum_{i=1}^{q} \alpha_{i}\left|\frac{r_{t-i}}{\sigma_{t-i}}\right|+\gamma_{i} \frac{r_{t-i}}{\sigma_{t-i}}\right) \\
& +\sum_{j=1}^{p} \beta_{j} \log \left(\sigma_{t-j}^{2}\right)
\end{aligned}
$$

In this model volatility asymmetrically reacts to good and bad news. In particular, the model includes asymmetric effects if some of the coefficients $\gamma_{i}$ are negative. The term in $\alpha_{i}$ represents a magnitude effect.

The conditional variance of an Asymmetric Power ARCH model, APARCH (p,q), [21] is defined as:

$$
\left.\sigma_{t}^{\delta}=\omega+\sum_{i=1}^{q} \alpha_{i}\left(\left|r_{t-i}\right|-\gamma_{i}\right)^{\delta}-\gamma_{i}\right)+\sum_{j=1}^{p} \beta_{j} \sigma_{t-j}^{\delta}
$$

where $\omega>0, \alpha_{\mathrm{i}} \geq 0, \beta_{\mathrm{j}} \geq 0, \delta>0,\left|\gamma_{\mathrm{i}}\right|<1$.

By adequately specifying the values for the coefficients, it is possible to obtain a variety of simpler GARCH type specifications as a special case of the APARCH model.

To account for the heavy tails effect we enlarge the class of models considering as possible choices for the distribution of the error term, $f\left(\mathrm{z}_{\mathrm{t}}\right)$, the Normal distribution and the Student's t-distribution.

When we need to select among many alternative model structures, we may not have a single model that outperforms, in terms of fitting and forecast accuracy, all the others. Many model evaluation criteria are suitable for pairwise-comparison and/or require the choice of a benchmark, that is not so obvious to select (i.e. [20], [30], [1]). In case we are interested in comparing more than two models and avoid the benchmark specification we may refer to the Model Confidence Set (MCS) by [33]. The objective of the MCS procedure is to determine a subset of models, $\mathrm{M} *$, that contains the best model with a given level of confidence, where best is defined in terms of any chosen criteria. The outcome of this approach is set of superior models, $M_{1-\alpha}^{*}$, that are not distinguishable from the best model across all the competing candidates.

\section{Empirical Analysis}

The volatility of the cryptocurrencies time series has been estimated through different conditional heteroschedasticity models variations. The empirical analysis is carried out considering a set, $\mathrm{M}$, of 10 candidate models. Namely, we consider the GARCH, GJR, TARCH, EGARCH and APARCH models labelled as M1, M10. M1 to M5 use the Normal distribution for the error term, and M5 to M10 the Student's t-distribution. Table 3 reports the insample estimates for the ten model specifications to M1,.., M10. As model evaluation statistics, we also include the AIC (Akaike Information Criterion) and BIC (Bayesian Information Criterion).

The reached results show a good performance for all the models at least in terms of significance of the parameters estimations. Looking at the presence of 
Table 3: Estimation results for the candidate models

\begin{tabular}{|c|c|c|c|c|c|c|c|c|c|c|}
\hline & M1 & M2 & M3 & M4 & M5 & M6 & M7 & M8 & M9 & M10 \\
\hline \multicolumn{11}{|l|}{ BTC } \\
\hline$\omega$ & $0.0003 * * *$ & 0.000 & $0.0001 * * *$ & $-0.7373 * * *$ & $0.0001 * * *$ & 1.0425 & 0.6304 & 1.4083 & -0.2127 & $1.7834 *$ \\
\hline$\alpha$ & $0.1605 * * *$ & $0.1594 * * *$ & $0.1672 * * *$ & $0.3088 * * *$ & $0.1703 * * *$ & $0.3048 * * *$ & $0.2640 * * *$ & $0.2207 * * *$ & $0.3465 * * *$ & $0.1985 * * *$ \\
\hline$\beta$ & $0.8078 * * *$ & $0.8098 * * *$ & $0.8172 * * *$ & $0.9215 * * *$ & $0.8166 * * *$ & $0.8695 * * *$ & $0.8859 * * *$ & $0.8890 * * *$ & $0.9931 * * *$ & $0.8885 * * *$ \\
\hline$\gamma$ & & -0.0190 & 0.0186 & -0.0085 & 0.0117 & & $-0.1476 * *$ & $-0.1924 * *$ & $0.0576 * *$ & $-0.2178 * *$ \\
\hline$\delta$ & & & & & $1.1203 * * *$ & & & & & $0.7818 * *$ \\
\hline AIC & -6372.8897 & -6371.0901 & -6382.98626 & -6382.52559 & -6381.32878 & -6827.27844 & -6831.75848 & -6852.52166 & -6845.01743 & -6851.56076 \\
\hline $\mathrm{BIC}$ & -6351.3093 & -6371.09011 & -6356.01072 & -6382.52559 & -6348.95813 & -6800.30291 & -6799.38784 & -6840.51184 & -6812.64678 & -6813.79500 \\
\hline \multicolumn{11}{|l|}{ ETH } \\
\hline$\omega$ & $0.0003 * * *$ & $0.0003 * * *$ & $0.0003 * * *$ & $-0.7446 * * *$ & $0.0003 * * *$ & $0.0002 * * *$ & $0.0002 * * *$ & $0.0002 * * *$ & $-0.5997 * * *$ & $0.0002 * *$ \\
\hline$\alpha$ & $0.1864 * * *$ & $0.1768 * * *$ & $0.1648 * * *$ & 0.1648 & $0.1680 * * *$ & $0.3245 * * *$ & $0.3321 * * *$ & $0.2454 * * *$ & $0.4253 * * *$ & $0.2670 * * *$ \\
\hline$\beta$ & $0.7328 * * *$ & $0.7426 * * *$ & $0.7943 * * *$ & $0.7943 * * *$ & $0.7907 * * *$ & $0.7473 * * *$ & $0.7440 * * *$ & $0.8010 * * *$ & $0.9380 * * *$ & $0.7911 * * *$ \\
\hline$\gamma$ & & -0.0520 & -0.0990 & -0.0990 & -0.0927 & & 0.0249 & 0.0096 & 0.0008 & 0.0173 \\
\hline$\delta$ & & & & & $1.0679 * * *$ & & & & & $1.1859 * *$ \\
\hline AIC & -4814.1280 & -4813.4726 & -4823.0509 & -4823.0509 & -4821.1282 & -5096.8319 & -5095.0421 & -5100.1469 & -5098.5110 & -5098.6594 \\
\hline $\mathrm{BIC}$ & -4792.5475 & -4786.4970 & -4796.0754 & -4796.0754 & -4788.7576 & -5069.8564 & -5062.6715 & -5067.7762 & -5066.1403 & -5060.8936 \\
\hline \multicolumn{11}{|l|}{ XLM } \\
\hline$\omega$ & $0.0003 * * *$ & $0.0003 * * *$ & $0.0004 * * *$ & $-0.8143 *$ & $0.0004 * * *$ & $0.0003 * * *$ & $0.0003 * * *$ & $0.0003 * * *$ & $-0.6451 * * *$ & $0.0003 * * *$ \\
\hline$\alpha$ & $0.2001 *$ & 0.1786 & $0.1906 * * *$ & $0.3613 * * *$ & $0.1899 * * *$ & $0.3660 * * *$ & $0.3609 * * *$ & $0.2732 * *$ & $0.4525 * *$ & $0.3017 * *$ \\
\hline$\beta$ & $0.7718 * * *$ & $0.7765 * * *$ & $0.7868 * * *$ & $0.8972 * * *$ & $0.7847 * * *$ & $0.7244 * * *$ & $0.7217 * * *$ & $0.7751 * * *$ & $0.9334 * * *$ & $0.7625 * * *$ \\
\hline$\gamma$ & & $-0.1347 * * *$ & $-0.2818 * * *$ & $0.0700 * * *$ & $-0.3058 * * *$ & & -0.0482 & $-0.1542 *$ & 0.0300 & -0.1144 \\
\hline$\delta$ & & & & & $0.8755 * * *$ & & & & & $1.2600 * * *$ \\
\hline AIC & -4419.1765 & -4424.5541 & -4440.4798 & -4428.1417 & -4438.7945 & -4920.2899 & -4919.0369 & -4922.7676 & -4917.5532 & -4921.9843 \\
\hline $\mathrm{BIC}$ & -4397.5961 & -4397.5786 & -4413.5042 & -4401.1662 & -4406.4239 & -4893.3144 & -4886.6663 & -4890.3970 & -4885.1826 & -4884.2185 \\
\hline \multicolumn{11}{|l|}{ XRP } \\
\hline$\omega$ & $0.0004 *$ & 0.0004 & $0.0006 * *$ & $-1.2292 * * *$ & 0.0006 & 0.0002 & 0.0003 & 0.0003 & $-0.7000 * * *$ & $0.0003 *$ \\
\hline$\alpha$ & $0.3095 *$ & 0.3058 & $0.3184 * * *$ & $0.4136 * * *$ & $0.3202 * * *$ & 0.9421 & 0.9857 & $0.5583 * *$ & $0.8849 * *$ & $0.4992 * *$ \\
\hline$\beta$ & $0.6388 * *$ & $0.6076 * * *$ & $0.6329 * * *$ & $0.8382 * * *$ & $0.6338 * * *$ & $0.7483 * * *$ & $0.7500 * * *$ & $0.7597 * * *$ & $0.9156 * * *$ & $0.7611 * * *$ \\
\hline$\gamma$ & & $-0.2030 * *$ & -0.3703 & $0.1435 * * *$ & -0.3361 & & 0.047 & -0.0225 & -0.0543 & -0.0417 \\
\hline$\delta$ & & & & & 1.1411 & & & & & $0.8605 * *$ \\
\hline AIC & -5082.9561 & -5103.0027 & -5111.6587 & -5052.1931 & -5110.0217 & -5778.7761 & -5777.5747 & -5793.2081 & -5775.3844 & -5791.6620 \\
\hline $\mathrm{BIC}$ & -5061.3757 & -5076.0271 & -5084.6831 & -5025.2175 & -5077.6511 & -5751.8006 & -5745.2040 & -5760.8374 & -5743.0137 & -5753.8963 \\
\hline
\end{tabular}

Notes: Sample period: 1 September 2015 - 14 February 2020. Number of daily observations: $1628 . *, * *$ and $* * *$ represent the significance at levels $10 \%, 5 \%$,

$1 \%$, respectively. 
asymmetric effect, given by a significant $\gamma$ coefficient, it is present for almost all the series, except for Ethereum which show a non-significative $\gamma$ for each of the considered model specifications. More in de- tails, for Bitcoin a significative $\gamma$ parameter can be observed for the model from M7 to M10 which include the t-distribution error term. For Stellar Lumens and Ripple the significance of the asymmetric coefficients appears in the model with a normal error specification, $z_{t} \sim N(0,1)$, namely for the model M2 to M5 for XLM and M2 and M4 for XRP. In addition, we can also note a significative $\gamma$ parameter at $10 \%$ for the model M8 estimated for Stellar Lumens. Furthermore, the negative sign of the leverage effect coefficient observed in most of the cases gives evidence of an inverted reaction of the volatility compared with the usual finding in stock market returns. This result is in line with the empirical evidence highlighted in the recent literature. [5] have observed this inverted asymmetric reaction, with positive shocks incrementing the volatility by more than negative ones, associating this effect to the trading activity of informed investor.

To avoid model uncertainty, we further compare the performance of the estimated models computing the
MCS that allow to select the set of best models from the $\mathrm{M}$ set of the 10 candidates. In the statistical evaluation with the MCS we use as proxies of the volatility measures the standard square returns (SQR) and the high-low corrected range (HL), which are unbiased proxies for the volatility. The number of bootstrapped samples for calculating the test statistic is 5000 and $\alpha$ is set to be equal to 0.25 . The loss function employed is the Mean Square Error (MSE) that can be defined robust to the proxy noise in the sense of [45]. The results of Table 4 reveal a clear tendency of the models estimated with the normality assumption on the error term to outperform their competitors, in particular for Bitcoin. Namely, the EGARCH enter the model confidence set the larger number of times supporting the asymmetric hypothesis. Those conclusions are also in line with previous analysis [34]. As expected, the MCS based on SQR and HL volatility measures are characterized by performances very similar. However, the HL seems to lead to more stable, in terms of numbers of models included, set of superior models.

Table 4: MCS compositions: p-values and models included.

\begin{tabular}{llccccccccccc}
\hline & & \multicolumn{2}{c}{ BTC } & \multicolumn{2}{c}{ ETH } & \multicolumn{2}{c}{ XLM } & \multicolumn{3}{c}{ XRP } \\
\hline & & \multicolumn{2}{c}{ SQR } & HL & SQR & HL & SQR & HL & SQR & HL & $n . m o d$ \\
M1 & GARCH & $0.2864 *$ & 0.2846 & $0.2846 *$ & 0.0554 & $0.3800 *$ & 0.1368 & $0.7624 *$ & $0.6432 *$ & 5 \\
M2 & GJR & $0.2864 *$ & 0.0056 & 0.0056 & 0.0554 & $0.3800 *$ & 0.1368 & $0.3844 *$ & $0.3838 *$ & 4 \\
M3 & TARCH & $0.6312 *$ & 0.0518 & $0.2846 *$ & 0.0554 & $0.3978 *$ & 0.1368 & $0.7624 *$ & $0.8488 *$ & 5 \\
M4 & EGARCH & $1.0000 *$ & $1.0000 *$ & 0.0056 & $1.0000 *$ & $0.3800 *$ & 0.1368 & $0.7624 *$ & $0.9654 *$ & 6 \\
M5 & APARCH & 0.2351 & 0.0158 & $0.2846 *$ & 0.0554 & $1.0000 *$ & $1.0000 *$ & $1.0000 *$ & $1.0000 *$ & 5 \\
M6 & GARCH-t & 0.1180 & 0.0428 & 0.0000 & 0.0022 & $0.3800 *$ & 0.1344 & $0.7080 *$ & $0.6432 *$ & 3 \\
M7 & GJR-t & 0.1622 & 0.0518 & $1.0000 *$ & 0.0022 & $0.3760 *$ & 0.1344 & $0.7080 *$ & $0.3838 *$ & 4 \\
M8 & TARCH-t & 0.0002 & 0.0126 & 0.0000 & 0.0006 & $0.3800 *$ & 0.1344 & $0.3478 *$ & $0.2532 *$ & 3 \\
M9 & EGARCH-t & 0.0002 & 0.0010 & $0.2846 *$ & 0.0000 & $0.3760 *$ & 0.1344 & $0.3230 *$ & 0.2332 & 3 \\
M10 & APARCH-t & 0.0004 & 0.0134 & 0.0000 & 0.0012 & $0.3800 *$ & 0.1344 & $0.3230 *$ & 0.2332 & 2 \\
\hline \# (MCS) & & 4 & 1 & 5 & 1 & 10 & 1 & 10 & 8 & \\
\hline
\end{tabular}

Notes: The table reports MCS p-values calculated including as proxy the squared returns (SQR) and he high-low corrected range (HL). Stars denote inclusion in the MCS at significance level $\alpha=0.25$. \#(MCS) is the size of the MCS, the number of models entering the best set for each cryptocurrency; n.mod denotes the number of times a given model enters the MCS. 


\section{Concluding remarks}

In this paper, we have investigated the opportunity to use different GARCH specifications for modelling volatility dynamics of cryptocurrency markets. The features of this market, characterized by heavy price fluctuations and sharp peaks, give evidence in favor of the employment of conditional heteroskedasticity models, originally proposed within stock markets context. Given the variety of modelling options, choosing a model specification represents a relevant source of uncertainty a practitioner has to deal with. Therefore, to evaluate and compare the volatility models performances and to select the set of superior models we realized on a formal calculation of the MCS with different settings. The analysis has been conducted considering ten GARCH structures estimated on four most popular cryptocurrencies in terms of their market capitalization: Bitcoin, Ethereum, Stellar and Ripple. The empirical findings confirmed the non-normality and heteroskedasticity of the cryptocurrencies' returns highlighted in previous empirical studies on the topic. Moreover, given the different specifications of each cryptocurrency, the comparison analysis shows that asymmetric volatility models, such as EGARCH models, are more often selected into the MCS as well as the models based on a normal distribution assumption for the error term.

The optimal GARCH-type specifications resulting by the analysis carried out can be further investigated by comparing the out-of-sample performances by means of economic loss functions such as risk management measures. This will be left to future research.

\section{Acknowledgements}

We would like to thank the Editor and the three anonymous Referees for their constructive comments and suggestions which led to improvements.

\section{References:}

[1] Amendola, A. and V. Candila (2016). Evaluation of volatility predictions in a var framework. Quantitative Finance 16(15), 695-709.

[2] Amendola, A. and G. Storti (2015). Model uncertainty and forecast combination in highdimensional multivariate volatility prediction. Journal of Forecasting 34(2), 83-91.

[3] Ardia, D.; Bluteau, K. R. M. (2019). Regime changes in bitcoin Garch volatility dynamics. Finance Research Letters 29, 266-271.
[4] Baur, D. G., T. Dimpfl, and K. Kuck (2018). Bitcoin, gold and the us dollar replication and extension. Finance Research Letters 25, 103-110.

[5] Baur, Dirk G.; Dimpfl, T. (2018). Asymmetric volatility in cryptocurrencies. Economics Letters 173, 148-151.

[6] Bauwens, L., C. Hafner, and L. S. (2012). Handbook of Volatility Models and Their Applications. Wiley.

[7] Bhme, R., N. Christin, B. Edelman, and T. Moore (2005). Bitcoin: economics, technology, and governance. Journal of Economic Perspectives 29, 213-238.

[8] Bollerslev, T. (1986). Generalized autoregressive conditional heteroskedasticity. Journal of Econometrics 31, 307-327.

[9] Bouri, E., P. Molnr, G. Azzi, and L. Roubaud, D.and Hagfors (2017). On the hedge and safe haven properties of bitcoin: is it really more than a diversifier? Finance Research Letters 20, 192- 198. [10] Bouri, E., P. Molnr, G. Azzi, and L. Roubaud, D.and Hagfors (2019). Cryptocurrencies and the downside risk in equity investments. Finance Research Letters 33, 1-14.

[11] Brauneis, A., R. Mestel, and E. Theissen (2020). What drives the liquidity of cryptocurrencies? a longterm analysis. Finance Research Letters in press.

[12] Catania, L., S. Grassi, and F. Ravazzolo (2019). Forecasting cryptocurrencies under model and parameter instability. International Journal of Forecasting 35, 485-501.

[13] Chan, Stephen; Chu, J. N. S. O. J. (2017). A statistical analysis of cryptocurrencies. Journal of Risk and Financial Management 10(12), 1-17.

[14] Choo, K.-K. R. (2015a). Cryptocurrency and virtual currency: Corruption and money laundering terrorism financing risks? Handbook of Digi- tal Currency Bitcoin, Innovation, Financial Instruments, and Big Data, 283-307.

[15] Choo, K.-K. R. (2015b). Is bitcoin a real currency. Handbook of Digital Currency Ch.2.

[16] Chowdhury, R., M. Arifur Rahman, M. Sohel Rahman, and M. M.R.C. (2000). An approach to predict and forecast the price of constituents and index of cryptocurrency using machine learning. Physica A: Statistical Mechanics and its Applications 551, 1-17.

[17] Conrad, Christian; Custovic, A. G. E. (2018). Long and short-term cryptocurrency volatility components: A Garch-Midas analysis. Journal of Risk and Financial Management 11, 23.

[18] Corbet, S., B. Lucey, A. Urquhart, and L. Yarovaya (2019). Cryptocurrencies as a financial asset: A systematic analysis. International Review of Financial Analysis 62, 182-199. 
[19] Corbet, S., B. Lucey, and L. Yarovaya (2018). Financial liberalization, stock markets and economic development. Finance Research Letters 26(0), 8188. [20] Diebold, F. X. and R. S. Mariano (1995). Comparing predictive accuracy. Journal of Business \& Economic Statistics 13(3), 253-263.

[21] Ding, Z., C. W. J. Granger, and R. F. Engle (1993). A long memory property of stock market returns and a new model. Journal of Empirical Finance 1(1), 83-106.

[22] Dyhrberg, A. (2016). Bitcoin, gold and the dollar. A Garch volatility analysis. Finance Research Letters 16, 85-92.

[23] ECB (2015). Virtual currency schemes - a further analysis. Technical report, European Central Bank, https://www.ecb.europa.eu.

[24] ECB (2019). Crypto-assets: Implications for financial stability, monetary policy, and payments and market infrastructures. Technical report, European Central Bank, https://www.ecb.europa.eu. [25] Engle, R. F. (1982). Autoregressive conditional heteroscedasticity with estimates of the variance of United Kingdom inflation. Econometrica 50, $987-$ 1007.

[26] Europol (2017). Europol 2017, Internet organised crime threat assessment. Technical report, https://www.europol.europa.eu/activitiesservices/main-reports/internet-organised-crimethreat-assessment-iocta-2017.

[27] Feng, W. and Y. Z. Z. Wang (2018). Can cryptocurrencies be a safe haven: a tail risk perspective analysis. Applied Economics 25, 47454762.

[28] Fry, J. and E. Cheah (2018). On bitcoin markets (in)efficiency and its evolution. Physica A: Statistical Mechanics and its Applications 203, 257-262.

[29] Fry, J. and E.-T. Cheah (2016). Negative bubbles and shocks in cryptocurrency markets. International Review of Financial Analysis 47, 343 352.

[30] Giacomini, R. and H. White (2006). Tests of conditional predictive ability. Econometrica 74(6), 1545-1578.

[31] Glosten, L. R., R. Jagannanthan, and D. E. Runkle (1993). On the relation between the expected value and the volatility of the nominal excess return on stocks. The Journal of Finance 48(5), 1779- 1801. [32] Hafner, C. M. (2018). Speculative bubbles in bitcoin markets? an empirical investigation into the fundamental value of bitcoin. Journal of Financial Econometrics, 1-17.

[33] Hansen, P. R., A. Lunde, and J. M. Nason (2011). The Model Confidence Set. Econometrica 79(2), 453-497.
[34] Hattori, T. (2019). A forecast comparison of volatility models using realized volatility: evidence from the bitcoin market. Applied Economics Letters. [35] Hencic, A. and C. Gouriroux (2015). Noncausal autoregressive model in application to bitcoin/USD exchange rates. Econometrics of Risk. Studies in Computational Intelligence 583, 17-40.

[36] Houben, R. and A. Snyers (2018). Cryptocurrencies and blockchain. legal context and implications for financial crime, money laundering and tax evasion. Technical report, IPOL - Policy Department for Economic, Scientific and Quality of Life Policies, European Parliament.

[37] Katsiampa, P. (2017). Volatility estimation for bitcoin: A comparison of Garch models. Economics Letters 158, 3-6.

[38] Koehuis, W., K. Tahar, and L. Nhien-An (2015). Bitcoin as an investment or speculative vehicle? a first look. Applied Economics Letters 22, 30-34.

[39] Koehuis, W., K. Tahar, and L. Nhien-An (2020). Forensic analysis of privacy-oriented cryptocurrencies, forensic science international: Digital investigation. Forensic Science International: Digital Investigation in press.

[40] Maese, V. A., A. W. Avery, B. A. Naftalis, S. P. Wink, and Y. D. Valdez (2016). Cryptocurrency: A primer. Banking Law Journal, 468-471.

[41] Mauro, C., E. Kumar, and L. S. R. Chhagan (2018). A survey on security and privacy issues of bitcoin. IEEE Communications Surveys \& Tutorials 20, 3416-3452.

[42] Mersch, Y. (2018). Member of the executive board of the ECB, official monetary and financial institutions forum, London, 2018, Virtual or virtueless? the evolution of money in the digital age. Technical report, official monetary and Financial Institutions Forum, London, 2018, https://www.ecb.europa.eu/press/key/date/2018/

[43] Nelson, D. B. (1991). Conditional heteroskedasticity in asset returns: A new approach. Econometrica 59, 347-370.

[44] Ngunyi, A.and Mundia, S. and C. Omari (2019). Modelling volatility dynamics of cryptocurrencies using Garch models. Journal of Mathematical Finance 9, 591-615.

[45] Patton, A. (2011). Volatility forecast comparison using imperfect volatility proxies. Journal of Econometrics 160(1), 246-256.

[46] Peng, Y., P. Albuquerque, J. M. Camboim de Sana, J. A. Padula, and M. R. Montenegro (2018). The best of two worlds: Forecasting high frequency volatility for cryptocurrencies and traditional currencies with support vector regression. Expert Systems with Applications 97, 177-192. 
[47] Rabemananjara, R. and J. M. Zako1an (1993). Threshold ARCH models and asymmetries in volatility. Journal of Applied Econometrics 8(1), 3149.

[48] Segnon, M. and S. Bekiros (2019). Forecasting volatility in cryptocurrency markets. CQE Working Papers 7919, Center for Quantitative Economics (CQE), University of Muenster.

[49] Selgin, G. (2015). Synthetic commodity money. Journal of Financial Stability 17, $92-99$.

[50] Stavroyiannis, S. (2018). Value-at-risk and related measures for the bitcoin. Journal of Risk Finance 19, 127-136.

[51] Tschorsch, F. and B. Scheuermann (2016). Bitcoin and beyond: A technical survey on decentralized digital currencies. IEEE Communications Surveys \& Tutorials 3, 2084-2123.

[52] Urquhart, A. and H. Zhang (2019). Is bitcoin a hedge or safe haven for currencies? An intraday analysis. International Review of Financial Analysis 63, 49-57.

[53] Chohan U.W. (2019), Are Stable Coins Stable? Notes on the 21st Century (CBRi), $10.2139 /$ ssrn.3326823

[54] Wang, G., M. Xin-yu, and W. Haoyu (2020). Are stablecoins truly diversifiers, hedges, or safe havens against traditional cryptocurrencies as their name suggests? Research in International Business and Finance 54, 1-18.

○

]

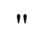

]
प

\&UHDMH\&RP P RQVS WMEXWRQ

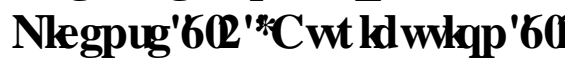

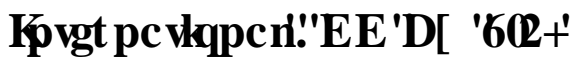

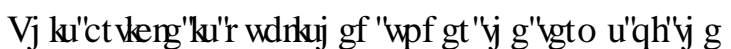

\&UDWXH\&RP P RQVS WUEXWRQ//FHQHID

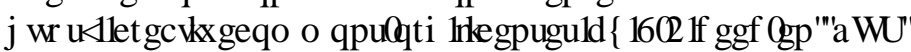

